\title{
Pathology of Gliomas and the Role Immunotherapy Plays in Combatting Glioma Proliferation
}

\author{
Divya Kothakapa $^{1}$ and Anush Swaminathan ${ }^{2 \#}$ \\ ${ }^{1}$ Great Valley High School, Malvern, PA, USA \\ ${ }^{2}$ Yale University, New Haven, CT, USA \\ "Advisor
}

$\underline{\text { ABSTRACT }}$

Glioma, a type of tumor arising from the brain and/or spinal cord, is a relatively broad term, encompassing three main types of glial cell tumors: astrocytoma, ependymoma, and oligodendroglioma. A prognosis for a glioma can often be extremely poor, with an overall survival rate of 54.84\%(1). With around 20,000 gliomas being diagnosed each year, it is imperative that effective treatments are developed for management of these tumors(2). A popular treatment pathway for the treatment of gliomas is surgery along with chemo-radiation therapy such as Temozolomide. This current treatment plan, though, has a moderate effect on lengthening glioma patients' prognoses. The average survival of this plan is 3 to 6 months (3). New treatments in the field of immunotherapies may have promising effects for glioma patients' prognoses. Arising developments in immunotherapy glioma treatment and management is an important field to investigate to further improve the effectivity of cancer treatment for glioma patients. Certain immunotherapies in trials have proven to make impactful improvements to glioma prognoses, one trial showing an increase of 2 months in median survival(4). This literature review will focus on the pathology and characteristics of the proliferation of malignant glioma cells and the role immunotherapy plays in combatting glioma proliferation.

\section{Pathology and Genetics of Gliomas}

Gliomas are a type of tumor that begins in glial cells of the brain and/or spinal cord. Subtypes of gliomas are based on the specific type of glial cell the neurological tumor originates from. The three types of glial cells that glioma tumors arise from are astrocytes, oligodendrocytes, and ependymal cells. Astrocytes look like a sun shaped cell with many dendrites sticking out of it. Their main role is to assist with blood flow in the brain, produce neurotransmitters, and send mitochondria to neurons(5). Oligodendrocytes look more like a pin wheel with their long and attachable dendrites. They are in charge of myelinating the axons of neurons, ensuring the quick neural processes(6). Lastly, ependymal cells have a rectangular shape and play a role in the production and transfer of cerebrospinal fluid(7). All of these glial cells are vital to the function and support of neurons, maintaining homeostasis of the central and peripheral nervous system.

Diagnoses of gliomas characterize them into grades from I to IV, formerly based on glial cell origination but now based on molecular and genetic characteristics. Low grade gliomas (Grade II) frequently progress to high grade gliomas (Grade III, IV), around 70\%(8). Genetic alterations of gliomas are one of the factors used to classify glioma grades. Multiple genetic mutations have been found to be related or correlated to the proliferation of malignant glioma cells. The pathological characterization of low grade II gliomas with a lack of clear border between the tumor and neighboring cells, as well as nuclear atypia(9). Higher grade gliomas have necrosis and marked nuclear atypia; thrombi, blood clots, are also frequently present in blood vessels around and in the tumor(9).

\section{LOH of $1 p$ and $19 q$}


Loss of the $1 \mathrm{p}$ and $19 \mathrm{q}$ chromosomes and chromosomal region $(\mathrm{LOH})$ are a known mutation related to the metastasis of gliomas, specifically oligodendrogliomas(10). This specific chromosomal mutation may also be indicative of favorable chemotherapy treatment results. A probable reason for this chemotherapy sensitivity is the specific special cell environment of the local environment and the supply of growth vigor(11). Another possible reason for the correlation of LOH 1p and 19q and chemotherapy sensitivity is the positive correlation between the chromosomes and the Sox 17 protein. The Sox 17 protein has been linked to decreased tumor cell differentiation and metastasis. The expression of Sox 17 leads to reduced alkylating agent resistance, and chemotherapy, being an alkylating agent, is able to increase its effectivity at targeting and damaging cancer cells' DNA. When LOH of 1p and 19q chromosomes occurs, there is an overexpression of genes related to the production of neurons(12). Conversely, when there is not a LOH of $1 p$ and $19 q$ chromosomes, there is an overexpression of immune response related genes. Therefore, when these gene sites are properly functioning, an adequate immune response is able to be activated by the immune system to fight against the proliferation of malignant cells, but when these genes fail or mutate, the body's immune system is unable to respond with enough effect and strength to prevent the further growth of the tumor. This finding leads to the interpretation that a loss of $1 \mathrm{p}$ and $19 \mathrm{q}$ are correlated to the inhibition of immune response and temper the effectivity of the immune system's ability to find against tumor cells, allowing for cancer cells to grow unchecked and undetected. There is a higher frequency of parietal lobe and frontal lobe glioma when there is a LOH of the 1p and 19q chromosomes, though the reason is unclear(13).

\section{EGFR}

The epidermal growth factor receptor on chromosome 7 is another known site that, if mutated, is correlated to the growth of malignant glioma cells(14); this specific mutation is found in $60 \%$ of gliomas(15). EGFR is a transmembrane glycoprotein that regulates the EGFR signaling pathways for cell growth, so when a mutation occurs at this receptor, there is activation of the signaling pathways leading to unbridled cell proliferation of these malignant gliomas(16). When the EGFR gene is functional, there is a proper moderated cell growth process, but because of mutant gene, the EGFR pathway becomes unregulated, with cell growth occurring continuously. This mutation occurs because of deletion in the DNA sequence of $801 \mathrm{bp}(17)$. When a mutation of EGFR occurs, it essentially continuously turns on the growth factor leading to continuous cell growth.

\section{9p and CDKN2A}

Deletion of the $9 \mathrm{p}$ is an important chromosomal mutation in the growth of gliomas as it results in the downregulation of CDKN2A, an integral tumor-suppressor gene (10) and occurs in 40\% of Grade IV gliomas (6). CDKN2A, normally, codes for the tumor-suppressor p16 and p14 proteins. When a mutation occurs in the CDKN2A, these proteins are not properly produced and the cell cycle regulation of preventing growth past the G1 cell cycle checkpoint does not occur because of the impaired proteins(18). A downregulation of this gene disrupts the normal regulatory cell cycle and the CDKN2A gene no longer negatively regulates cell growth, leading to rapid proliferation.

\section{$\mathrm{Rb}$ and P53}

Additionally, early mutations of the $\mathrm{Rb}$ and P53, both regulatory pathways of the $\mathrm{G} 1$ to $\mathrm{S}$ phase of the cell cycle, occur in low-grade gliomas, causing them to progress to higher grade gliomas (6). This mutation is critical to oncogenesis as the specific checkpoint between $\mathrm{G} 1$ to $\mathrm{S}$ dictates if the cell continues to proliferate. The specific Rb and p53 proteins negatively regulate this checkpoint, preventing cells from entering into the S phase(19). With this mutation, the regulatory pathway is altered, allowing for cells to continue to proliferate unregulated. Specific combinations of genetic mutations rather than single mutations, including those stated before, are thought to be the cause of gliomas-derived from the multiple hit hypothesis(20). The multiple hit hypothesis theorizes that malignant production of cells can not 
occur with singular gene mutations, but rather multiple different mutations occurring at the same time. This occurs because the DNA sequence incorporates redundant genes, so that if a single gene mutates, often other gene will be able to accommodate, but if there are multiple points of mutations, the body is not able to compensate and results in the uncheck growth of tumors.

Classification of primary and secondary gliomas is also based on the genetic alterations of the tumor. Primary tumors mainly show mutations in such chromosomal sites stated above, such as the EGFR, PTEN, and CDKN2A gene(21). Secondary gliomas frequently arise from previously low-grade gliomas, while primary gliomas grow rapidly de novo. Structurally, these two types of gliomas are somewhat similar, while their genetic makeup and components is the main difference. Secondary gliomas frequently have TP53 genetic mutations, indicating that a difference in genetic mutations may cause a different classification of glioma. The amplification of EGFR pathway of primary glioma mutations includes the mutated amplicons of the receptor, often with the deletions of variant 3's exons from 2 through 7(22). The increased activity of EGFR, a protein instrumental in the positive regulation of the cell growth process, results in decreased apoptosis and cell proliferation, as PI3K, enzyme that promotes cell survival and proliferation, successfully binds to PIP3 on the receptor's membrane. This binding then releases AKT and mTOR effector molecules, continuing the PI3K/AKT pathway that promotes cell growth and survival, increasing cell proliferation and reducing apoptosis. This mutation also results in the downregulation of p27, a tumor suppresor molecule that aims to slow down the cell cycle process, which increases the cell proliferation.

\section{Traditional Treatments for Gliomas}

Chemotherapy has been a standard part of the glioma treatment plan, often used after a surgical procedure to ensure tumor cell death. It has remained the popular standard of care because of it being one of the first, if not the first, drug to be an adequate treatment against cancer. It has also been the standard of care because of its ability to target cells away from the original tumor site, in case of metastasis; this ability is not found in other prevalent treatment types such as surgery or radiation, which are local treatments(23). Surgical removal of tumor masses is a another frequent treatment pathway, often used adjacently with an anti-cancer drug, such as chemotherapy. Removing the glioma surgically is done to ensure that a histological diagnosis of the tumor is done, as well as to remove large amounts of the tumor to reduce the overall tumor size. The most frequent surgical procedure for glioma treatment is a standard craniotomy approach. Patients are put under anesthesia and an incision is made through the skull, over the tumor location(24). Then, resection occurs, when pieces of tumor tissue removed from the skull. This treatment approach results in an 12-15 month extension in glioma patient survival(24). Only a select type of Glioma patient is able to receive a surgical reduction, though. Patients must have a KPS (Karnosky Performance Scale) index of above 70 in order to qualify for surgery(24) This index measures the patient's amount of functional impairment before a surgery to predict the level of improvement of a patient after surgery and level of function the patient will have post-operation(24). The latter is an important factor since functional ability often is further decreased after surgery, so it is critical to ensure that surgery does not further worsen the patient's ability and quality of life. Though, because of this requirement, a number of glioma patients are not qualified to undergo this surgical removal of tumors and must rely on other nonsurgical methods including, but not limited to, chemotherapy and radiation. The most common chemotherapy used for newly diagnosed tumors and after surgery is temozolomide chemotherapy. This treatment is frequently used for low grade tumors I and II. Temozolomide chemotherapy is characterized as an alkylating agent, attaching alkyl groups to the guanine base in cancer cells' DNA and causing cross bridges in the DNA strands that prevent the separation of the helix. This then prevents the proper transcription of DNA to RNA, thereby inhibiting the cancer cell's proliferation and protein synthesis. This treatment approach, though, does not prevent cancer recurrence, when cancer cells begin to proliferate after the removal of the tumor from the original site. Cancer recurrence can occur because some malignant cancer cells are left behind in the body, resulting in the re-accumulations and proliferation of gliomas(25). This treatment type has had a 90\% recurrence rate of gliomas(26). Another chemotherapy option for gliomas is Carmustine, a non-specific alkylating agent. Carmustine can either be administered via infusion or through a Carmustine wafer, a 
concentrated drug implant placed directly in the tumor site during surgery(27). Carmustine has a similar mechanism of action to Temozolomide treatment, as Carmustine causes cross bridges in DNA and RNA and inhibits from cancerous cells to further proliferate(28). Carmustine, alike to Temozolomide, also triggers the production of 06-methylguanine which has been linked to triggering apoptosis in cancer cells(29).

A critical issue with the current standard care plan of chemotherapies is that it leaves a large possibility for cancer reoccurrence and is not equipped with the ability to prevent from remaining cancer cells to repopulate; prevention of the recurrence of gliomas should be critical aspect of the treatment plan. The design of chemotherapy treatments may inhibit the drug's ability to have long-term and constant effectivity on cancer cell death. Chemotherapy is formulated to promote apoptosis in the glioma cancer cells while the treatment is still in the body; temozolomide chemotherapy, the standard treatment, is administered orally(30). This design gives chemotherapy a "short term" effect on tumors, restricted by the frequency and time since a dosage of the treatment has been administered. Longer term treatments that allow for anti-cancer effects and defense against malignant cells would contribute to the reduction of glioma recurrence rate. The side effects of these chemotherapies are also challenging to the quality of life and function of patients being administered them. As a result of chemotherapy, poor appetite and painful mouth sores ail patients, leading to poor nutritional status and further risk of infection to these severely immunocompromised patients(31). Fatigue and depression have been correlated to undergoing chemotherapy treatment, causing patients to have to endure both physical pain and symptoms, as well as psychological discomfort(31). These side effects can have debilitating consequences as they expose patients to an increased chance of complications and may cause lasting effects on patients' mental health.

Radiotherapy is another leading treatment for glioma tumors. The administration of radiotherapy involves dosages of radiation that damage the DNA the cancer cells, which, then, inhibits the proper transcription of RNA and prevents proliferation of cells. Radiotherapy is able to do this, by one, causing direct damage to cell DNA, making breaks in the helixes, and by also activating $\mathrm{H} 20$ molecules and other reactive molecules that will damage the DNA (32) as superoxide, hydroxyl radical, and hydrogen peroxide(33). ROS trigger cell damage in cancer and non-cancer cells by interfering with the components of the cell's DNA, for example, by removing H atoms from the thymine base's methyl group(34). The challenge with radiotherapy is finding the proper relationship between dosage amount and toxicity. There is a rationale for the beneficial effects of escalating dosage amounts past the 60 Gray (Gy), though there is an issue with the amount of neurologic toxicity this large of dosage may cause (35): Neurologic toxicity can result in a number of symptoms including seizure, paralysis, and dyskinesia (36). The bodily stresses of radiation may also cause neurodegeneration because of the radiation triggering gliosis, the rapid proliferation of glial cells that can result in scars on brain tissue, and damage to neurons and glia(36). Furthermore, the challenge of radiotherapy is also extended to the fact that even if the radiotherapy is increased to the tolerable levels of $60 \mathrm{~Gy}$, there is an $85 \%$ recurrence in glioma tumors after that radiotherapy dosage(35). This shows that even with increased radiotherapy levels, past standard but endurable levels, to a point that may cause damaging neurotoxicity, radiotherapy still largely results in recurrent gliomas and with no long-term aid in fighting the re-accumulation of malignant cells.

\section{Overview of Immunotherapy}

The immune system is the vital component that is utilized and manipulated to maximize effectivity of immunotherapies. The immune system's function is to formulate a response to pathogenic presences in the body and to fight against toxic or foreign substances(37). A large component of the immune response of the immune system are the white blood cells. White blood cell are in charge of detecting foreign substances in the body and then engaging an immune response on that foreign substance. There are multiple types of white blood cells, including the lymphocyte T-cells. T-cells are a vital aspect of the adaptive immune system, the system that tailors immune responses to certain substances. Having a effective T-cells within the body that are properly able to detect and attack cancer cells is imperative to reducing the proliferation of tumors. When cancerous tumors begin to form, cytotoxic immune cells ((NK) and CD8 ${ }^{+} \mathrm{T}$ cells $)$ 
target and kill immunogenic cells, which happen to play a role in provoking an immune response from the body(38). The immune system's inadequate response to the tumor cells and the cancerous cells' ability to go undetected by the immune system because of the elimination of immunogenic cancer cells promotes the proliferation of tumors.

Immunotherapies are designed to utilize and maximize the effectivity of the body's immune system to activate potent antitumor responses. There are different ways an immunotherapy is able to manipulate the immune system's response to amplify it; the three main types being immune checkpoint blockade, cancer vaccines, and $\mathrm{T}$ cell transfer therapy. The immune checkpoint blockade manner of immunotherapies targets certain cell receptors on cells to achieve a favorable activity from T cells. For example, certain receptors on $\mathrm{T}$ cells have been proven to contribute to the growth of malignant tumors, so immune checkpoint activity specifically targets these receptors to dampen their activity, thereby allowing for a more effective antitumor response from T cells. Secondly, cancer vaccines are less of a treatment and more of a preventative immunotherapy. These cancer vaccines have been formulated by administering tumor cells with an adjuvant to invoke an immune response. Lastly, adoptive T cell therapy was explored as a means of immunotherapy treatment. Within this treatment type, there are many different approaches to the procedure of harvesting and transferring T-cells to take advantage of these cells' abilities. One type is tumor infiltrating lymphocyte therapy (TIL). This method utilizes T-cells that have already infiltrated the tumor since they are already reactive to the tumor cells' antigens(39). Once these tumor infiltrating T-cells are isolated from the body, they are harvested in vitro to grow their count. These cells are then infused back into the body. The main purpose of this approach is to expand the number of T-cells and ensure that the T-cells in the body are activated to respond to the cancer cells. Some patients, though, may not have tumor infiltrating $\mathrm{T}$-cells that are already reactive to cancer antigens, so engineered $\mathrm{T}$ cell receptor therapy may be a beneficial approach. T-cells, not already activated to the tumor, are isolated from the body, and then a new receptor is engineered on to these cells that are specific to the tumor's antigens(40). Another prominent adoptive t-cell therapy approach is chimeric antigen receptor therapy (CAR). This approach also engineers new tumor-specific receptors onto T-cell, but CAR T-cell therapy does not rely on MHC, a complex that codes for surface proteins. Chimeric antigen receptors only need to be able to recognize the antigens present on cell surfaces.

\section{Immunotherapies specific to gliomas}

Immunotherapies have been a growing presence in the treatment field for glioma treatment. Its ability to allow for long term immune response effects shows is a promising pathway for glioma treatment. Bevacizumab, a monoclonal antibody targeting vascular endothelial growth factor, has been a developing and up and coming immunotherapy used for the treatment of glioblastomas. This therapy works by binding to the VEGF, preventing the growth factor's ability to bind with VEGFR-1 or VEGFR-2(41). This binding of VEGF results in the reduction of growth of tumor blood vessels, thereby limiting the glioma's ability to access blood supplies.

There are other promising pathways to tumor growth inhibition by immunotherapies. Through the immune checkpoint blockade technique, robust anti-tumor responses can be activated by targeting immune system receptors, molecules, and enzymes such as, CTLA-4 and PD-L1. PD-L1 is an immune system check point that plays an integral in apoptosis. This receptor is frequently activated in glioma cells and allows for cell growth and survival of the tumor cells. Immunotherapies specifically targeting this receptor have shown favorable effects at reducing the immunosuppressive effects of the expression of PD-L1. When the receptor was blocked, results showed that the immune system's natural killer cell groups, which would normally be blocked by PD-L1, were able to survive for 15 days longer than a control group without PD-L1 blockers(42). These natural killer groups 'receptor binds to NKG2DL, a NK gene on the tumor surface, which then causes the activation of NK cells and allows them to begin fighting the cancer cells(43). The immunotherapy Nivolumab utilizes the checkpoint blocker effect on PD-L1 to inhibit the growth of the tumor by increasing the survival rate of NK cells and increasing the impact of the immune response.

CTLA-4 is another receptor that inhibits the effectivity of the immune response on a glioma. When this receptor is bound with a protein, it inhibits immune cells' ability to kill other cells, thereby blocking the ability for the immune response to kill cancer cells. This receptor's expression is directly correlated to the presence of gliomas, as 
well as the growth of them. CTLA-4 and CD28 both have the same ligands, though CTLA-4 attracts the ligands much more than does CD28, resulting in ligands more often binding with CTLA-4 and being able to frequently activate. This receptor is actively preventing the ability for the CD28 receptor from activating. This is an impactful inhibition since the CD28 receptor plays a critical role in activating and promoting the survival of T cells. Targeting this receptor has been revealed to be an effective pathway of increasing glioma cell death and prolonging glioma prognosis; the immunotherapy ipilimumab aims to target CTLA-4 to for the increased effectivity of the immune response. When both these types of immune checkpoint blockade immunotherapies are administered together, the immune response is even more robust with $75 \%$ of murine glioblastomas being cured with this collective treatment plan (44).

\section{Future of Immunotherapies as treatment for Gliomas}

Immunotherapy research and development has given insight into what future applications of immunotherapies may be like and the direction the field is moving in. One promising development in immunotherapies has been the technique of adoptive lymphocyte transfer. This process includes the growing and activating of immune system molecules, which are then transferred back into the patient's body(45). This technique, then, branched into chimeric antigen receptor $\mathrm{T}$ cells. This technique artificially produces immune system receptors that can target specific proteins on the tumor surface. These CAR T cells are grown in populations and imbedded back into the patient's body. Another promising targeted drug, used in cases when a tumor reoccurs, is bevacizumab(46). Bevacizumab singularly binds to certain VEGFs (vascular endothelial growth factor), which plays a vital role in the production of blood vessels, thereby limiting these proteins to bind to tyrosine kinase receptors on the cell surface and inhibiting the growth of blood vessels to tumors(47). Another VEGF focused treatment type targets the growth factor and utilizes ectopic expression of VEGF to promotes immunosurveillance of gliomas(48). A study has found that VEGF-C expression leads to an increased level of lymphatic drainage; increased lymphatic drainage then increases the body's immunosurveillance, the body's ability to detect foreign substances and pathways(48). Such a finding would reveal that promoting increased lymphatic drainage would increase the effectivity of other immunotherapy drugs, such as checkpoint inhibitors, by making the immune system keener to detecting specifically cancer cells as foreign substances and targeting them. Lymphatic drainage is linked to the treatment of cancer cells because of its processes. During lymphatic drainage, a fluid called lymph that contains T-cells flows through the body's blood vessels and enters into cells and tissue(49). The fluid provides nutrients to cells and then collects toxins and waste, such as cancer cells from the tissue. This fluid then travels to the lymph glands where toxins are filtered from the lymph(49). This drainage process may be indicative of the reason for increased lymphatic drainage allowing the immune system to have a more effective and continuous immune detection and response to cancer cells and larger immunosurveillance, as increased drainage results in the increased circulation of T-cell containing lymph fluid.

\section{Acknowledgements}

Thank you to my advisor for the continued help in writing and discussing this paper and advising me in the overall process of research for the paper.

\section{References}

Wang, J., Hu, G., \& Quan, X. (2019). Analysis of the Factors Affecting the Prognosis of Glioma Patients. Open medicine (Warsaw, Poland), 14, 331-335. https://doi.org/10.1515/med-2019-0031

Mesfin FB, Al-Dhahir MA. Gliomas. [Updated 2020 Aug 10]. In: StatPearls [Internet]. Treasure Island (FL): StatPearls Publishing; 2020 Jan-. Available from: https://www.ncbi.nlm.nih.gov/books/NBK441874/ 
Chowdhary, M. M., Ene, C. I., \& Silbergeld, D. L. (2015). Treatment of Gliomas: How did we get here?. Surgical neurology international, 6(Suppl 1), S85-S88. https://doi.org/10.4103/2152-7806.151348

Desai, R., Suryadevara, C. M., Batich, K. A., Farber, S. H., Sanchez-Perez, L., \& Sampson, J. H. (2016). Emerging immunotherapies for glioblastoma. Expert opinion on emerging drugs, 21(2), 133-145.

https://doi.org/10.1080/14728214.2016.1186643

Kim, Y., Park, J., \& Choi, Y. K. (2019). The Role of Astrocytes in the Central Nervous System Focused on BK Channel and Heme Oxygenase Metabolites: A Review. Antioxidants (Basel, Switzerland), 8(5), 121. https://doi.org/10.3390/antiox8050121

Bradl, M., \& Lassmann, H. (2010). Oligodendrocytes: biology and pathology. Acta neuropathologica, 119(1), 3753. https://doi.org/10.1007/s00401-009-0601-5

Spassky N, Merkle FT, Flames N, Tramontin AD, García-Verdugo JM, Alvarez-Buylla A. Adult ependymal cells are postmitotic and are derived from radial glial cells during embryogenesis. J Neurosci. 2005 Jan 5;25(1):10-8. doi: 10.1523/JNEUROSCI.1108-04.2005. PMID: 15634762; PMCID: PMC6725217.

Altieri, R., Agnoletti, A., Quattrucci, F., Garbossa, D., Calamo Specchia, F. M., Bozzaro, M., Fornaro, R., Mencarani, C., Lanotte, M., Spaziante, R., \& Ducati, A. (2014). Molecular biology of gliomas: present and future challenges. Translational medicine @ UniSa, 10, 29-37.

Vigneswaran, K., Neill, S., \& Hadjipanayis, C. G. (2015). Beyond the World Health Organization grading of infiltrating gliomas: advances in the molecular genetics of glioma classification. Annals of translational medicine, 3(7), 95. https://doi.org/10.3978/j.issn.2305-5839.2015.03.57

Zhao, J., Ma, W., \& Zhao, H. (2014). Loss of heterozygosity 1p/19q and survival in glioma: a meta-analysis. Neurooncology, 16(1), 103-112. https://doi.org/10.1093/neuonc/not145

2 Li, J., Miao, N., Liu, M., Cui, W., Liu, X., Li, X., Shi, X., Qing, S., Ma, Y., Zhang, W., \& Biekemituofu, H. (2014). Clinical significance of chromosome 1p/19q loss of heterozygosity and Sox 17 expression in oligodendrogliomas. International journal of clinical and experimental pathology, 7(12), 8609-8615.

Ferrer-Luna, R., Mata, M., Núñez,L., Calvar, J., Dasí, F., Arias, E., Piquer, J., Cerdá-Nicolás, M., Taratuto, A. L., Sevlever, G., Celda, B., \& Martinetto, H. (2009). Loss of heterozygosity at 1p-19q induces a global change in oligodendroglial tumor gene expression. Journal of neurooncology, 95(3), 343-354.

https://doi.org/10.1007/s11060-009-9944-y

Ren, X., Cui, X., Lin, S., Wang, J., Jiang, Z., Sui, D., Li, J., \& Wang, Z. (2012). Co-deletion of chromosome 1p/19q and IDH1/2 mutation in glioma subsets of brain tumors in Chinese patients. PloS one, 7(3), e32764. https://doi.org/10.1371/journal.pone.0032764

Saadeh, F. S., Mahfouz, R., \& Assi, H. I. (2018). EGFR as a clinical marker in glioblastomas and other gliomas. The International journal of biological markers, 33(1), 22-32. https://doi.org/10.5301/ijbm.5000301

Gladson, C. L., Prayson, R. A., \& Liu, W. M. (2010). The pathobiology of glioma tumors. Annual review of pathology, 5, 33-50. https://doi.org/10.1146/annurev-pathol-121808-102109 
Bethune, G., Bethune, D., Ridgway, N., \& Xu, Z. (2010). Epidermal growth factor receptor (EGFR) in lung cancer: an ov erview and update. Journal of thoracic disease, 2(1), 48-51.

Xu, H., Zong, H., Ma, C., Ming, X., Shang, M., Li, K., He, X., Du, H., \& Cao, L. (2017). Epidermal growth factor receptor in glioblastoma. Oncology letters, 14(1), 512-516. https://doi.org/10.3892/ol.2017.6221

McWilliams, R. R., Wieben, E. D., Rabe, K. G., Pedersen, K. S., Wu, Y., Sicotte, H., \& Petersen, G. M. (2011). Prevalence of CDKN2A mutations in pancreatic cancer patients: implications for genetic counseling. European journal of human genetics : EJHG, 19(4), 472-478. https://doi.org/10.1038/ejhg.2010.198

Giacinti, C., Giordano, A. RB and cell cycle progression. Oncogene 25, 5220-5227 (2006). https://doi.org/10.1038/sj.onc.1209615

Al Hajri, Q., Dash, S., Feng, Wc. et al. Identifying multi-hit carcinogenic gene combinations: Scaling up a weighted set cover algorithm using compressed binary matrix representation on a GPU. Sci Rep 10, 2022 (2020). https://doi.org/10.1038/s41598-020-58785-y

Appin, C. L., \& Brat, D. J. (2014). Molecular genetics of gliomas. Cancer journal (Sudbury, Mass.), 20(1), 66-72. https://doi.org/10.1097/PPO.0000000000000020

Ohgaki, H., \& Kleihues, P. (2007). Genetic pathways to primary and secondary glioblastoma. The American journal of pathology, 170(5), 1445-1453. https://doi.org/10.2353/ajpath.2007.070011

How Is Chemotherapy Used to Treat Cancer? (2019, November 19). Retrieved February 2, 2021, from https://www.cancer.org/treatment/treatments-and-side-effects/treatmenttypes/chemotherapy/how-is-chemotherapy-used-to-treatcancer.html\#references

Young, R. M., Jamshidi, A., Davis, G., \& Sherman, J. H. (2015, June). Current trends in the surgical management and treatment of adult glioblastoma. Retrieved from https://www.ncbi.nlm.nih.gov/pmc/articles/PMC4481356/ https://www.cancer.gov/types/recurrentcancer\#: : text $=$ Why $\% 20$ Cancer $\% 20$ Comes $\% 20$ Back,up $\% 20 \mathrm{in} \% 20$ follow $\% 2$ Dup $\% 20$ tests.

Roy, S., Lahiri, D., Maji, T., \& Biswas, J. (2015). Recurrent Glioblastoma: Where we stand. South Asian journal of cancer, 4(4), 163-173. https://doi.org/10.4103/2278-330X.175953 (Retraction published South Asian J Cancer. 2017 Oct-Dec;6(4):153)

Garside R, Pitt M, Anderson R, et al. The effectiveness and cost-effectiveness of carmustine implants and temozolomide for the treatment of newly diagnosed high-grade glioma: a systematic review and economic evaluation. 2007. In: NIHR Health Technology Assessment programme: Executive Summaries. Southampton (UK): NIHR Journals Library; 2003-. Available from: https://www.ncbi.nlm.nih.gov/books/NBK56869/

National Center for Biotechnology Information (2021). PubChem Compound Summary for CID 2578, Carmustine. Retrieved February 3, 2021 from https://pubchem.ncbi.nlm.nih.gov/compound/Carmustine. 
Papait R, Magrassi L, Rigamonti D, Cattaneo E. Temozolomide and carmustine cause large-scale heterochromatin reorganization in glioma cells. Biochem Biophys Res Commun. 2009 Feb 6;379(2):434-9. doi:

10.1016/j.bbrc.2008.12.091. Epub 2008 Dec 29. PMID: 19116135.

https://www.cancerresearch.org/blog/june-2016/difference-cancer-immunotherapy-andchemotherapy

Chui P. L. (2019). Cancer- and Chemotherapy-Related Symptoms and the Use of Complementary and Alternative Medicine. Asia-Pacific journal of oncology nursing, 6(1), 4-6. https://doi.org/10.4103/apjon.apjon 51

Baskar, R., Lee, K. A., Yeo, R., \& Yeoh, K. W. (2012). Cancer and radiation therapy: current advances and future directions. International journal of medical sciences, 9(3), 193-199. https://doi.org/10.7150/ijms.3635

Reisz, J. A., Bansal, N., Qian, J., Zhao, W., \& Furdui, C. M. (2014). Effects of ionizing radiation on biological molecules--mechanisms of damage and emerging methods of detection. Antioxidants \& redox signaling, 21(2), 260292. https://doi.org/10.1089/ars.2013.5489

Cooke, M. S., Evans, M. D., Dizdaroglu, M., \& Lunec, J. (2003, July 01). Oxidative DNA damage: Mechanisms, mutation, and dise ase. Retrieved from https://faseb.onlinelibrary.wiley.com/doi/full/10.1096/fj.02$\underline{0752 \mathrm{rev}}$

Dhermain F. (2014). Radiotherapy of high-grade gliomas: current standards and new concepts, innovations in imaging and radiotherapy, and new therapeutic approaches. Chinese journal of cancer, 33(1), 16-24.

https://doi.org/10.5732/cjc.013.10217

Smart D. (2017). Radiation Toxicity in the Central Nervous System: Mechanisms and Strategies for Injury Reduction. Seminars in radiation oncology, 27(4), 332-339. https://doi.org/10.1016/j.semradonc.2017.04.006

Chaplin D. D. (2010). Overview of the immune respons e. The Journal of allergy and clinical immunology, $125(2$ Suppl 2), S3-S23. https://doi.org/10.1016/j.jaci.2009.12.980

Gonzalez, H., Hagerling, C., \& Werb, Z. (2018). Roles of the immune system in cancer: from tumor initiation to metastatic pro gression. Genes \& development, 32(19-20), 1267-1284.

https://doi.org/10.1101/gad.314617.118

Tran, K. Q., Zhou, J., Durflinger, K. H., Langhan, M. M., Shelton, T. E., Wunderlich, J. R., Robbins, P. F., Rosenberg, S. A. , \& Dudley, M. E. (2008). Minimally cultured tumor-infiltrating lymphocytes display optimal characteristics for adoptive cell therapy. Journal of immunotherapy (Hagerstown, Md. : 1997), 31(8), $742-751$. https://doi.org/10.1097/CJI.0b013e31818403d5

Zhang, J., \& Wang, L. (2019). The Emerging World of TCR-T Cell Trials Against Cancer: A Systematic Review. Technology in cancer research \& treatment, 18, 1533033819831068. https://doi.org/10.1177/1533033819831068

https:/www.ncbi.nlm.nih.gov/pmc/articles/PMC3228024/\#: :text=Bevacizumab\%20acts\%20by\%20selectively\%20 binding,blood $\% 20$ supply\%2 0to\%20tumor\%20tissues. 
Shengchao Xu, Lu Tang, Xizhe Li, Fan Fan, Zhixiong Liu, Immunotherapy for glioma: Current management and future application, Cancer Letters, Volume 476, 2020, Pages 1-12, ISSN 0304-3835, https://doi.org/10.1016/j.canlet.2020.02.002.

(http://www.sciencedirect.com/science/article/pii/S0304383520300562) hes in glioma treatment.

Duan, S., Guo, W., Xu, Z. et al. Natural killer group 2D receptor and its ligands in cancer immune escape. Mol Cancer 18, 29 (2019). https://doi.org/10.1186/s12943-019-0956-8

D.A. Reardon, P.C. Gokhale, S.R. Klein, K.L. Ligon, S.J. Rodig, S.H. Ramkissoon, K.L. Jones, A.S. Conway, X. Liao, J. Zhou, P.Y. Wen, A.D. Van Den Abbeele, F.S. Hodi, L. Qin, N.E. Kohl, A.H. Sharpe, G. Dranoff, G.J. FreemanGlioblastoma eradication following immune checkpoint blockade in an orthotopic, immunocompetent model Canc. Immunol. Res., 4 (2016), pp. 124-135

Fecci, P. E., \& Sampson, J. H. (2019). The current state of immunotherapy for gliomas: an eye toward the future, Journal of Neurosurgery JNS, 131(3), 657-666. Retrieved Jan 26, 2021, from https://thejns.org/view/journals/j-neurosurg/131/3/article-p657.xml https://www.mskcc.org/cancer-care/types/glioma/glioma-treatment/chemotherapy-glioma

Kazazi-Hyseni, F., Beijnen, J. H., \& Schellens, J. H. (2010). Bevacizumab. The oncologist, 15(8), 819-825. https://doi.org/10.1634/theoncologist.2009-0317

Song, E., Mao, T., Dong, H. et al. VEGF-C-driven lymphatic drainage enables immunosurveillance of brain tumours. Nature 577, 689-694 (2020). https://doi.org/10.1038/s41586-019-1912-X

Padera, T. P., Meijer, E. F., \& Munn, L. L. (2016). The Lymphatic System in Disease Processe $\mathrm{s}$ and Cancer Progression. Annual review of biomedical engineering, 18, 125-158. https://doi.org/10.1146/annurevbioeng-112315-031200 